Published in final edited form as:

Neurourol Urodyn. 2017 June ; 36(5): 1270-1277. doi:10.1002/nau.23105.

\title{
Sacral neuromodulation of nociceptive bladder overactivity in cats
}

\author{
Zhaocun Zhang ${ }^{1,2}$, Jathin Bandari ${ }^{1}$, Utsav Bansal ${ }^{1}$, Bing Shen ${ }^{1}$, Jicheng Wang ${ }^{1}$, Vladimir \\ Lamm $^{1}$, James R. Roppolo ${ }^{3}$, William C. de Groat ${ }^{3}$, and Changfeng Tai ${ }^{1,3,{ }^{*}}$ \\ ${ }^{1}$ Department of Urology, University of Pittsburgh, Pittsburgh, Pennsylvania \\ 2Department of Urology, Qilu Hospital, Shandong University, Jinan, P.R. China \\ ${ }^{3}$ Department of Pharmacology and Chemical Biology, University of Pittsburgh, Pittsburgh, \\ Pennsylvania
}

\section{Abstract}

\begin{abstract}
AIMS-To investigate the effects of electrical stimulation of sacral dorsal/ventral roots on irritation-induced bladder overactivity, reveal possible different mechanisms under nociceptive bladder conditions, and establish a large animal model of sacral neuromodulation.

METHODS-Intravesical infusion of $0.5 \%$ acetic acid (AA) was used to irritate the bladder and induce bladder overactivity in cats under $a$-chloralose anesthesia. Electrical stimulation (5, 15, or $30 \mathrm{~Hz}$ ) was applied to individual S1-S3 dorsal or ventral roots at or below motor threshold intensity. Repeated cystometrograms (CMGs) were performed with/without the stimulation to determine the inhibition of bladder overactivity.
\end{abstract}

RESULTS-AA irritation induced bladder overactivity and significantly $(P<0.05)$ reduced the bladder capacity to $62.6 \pm 11.7 \%$ of control capacity measured during saline CMGs. At threshold intensity for inducing reflex twitching of the anal sphincter or toe, S1/S2 dorsal root stimulation at $5 \mathrm{~Hz}$ but not at 15 or $30 \mathrm{~Hz}$ inhibited bladder overactivity and significantly $(P<0.05)$ increased bladder capacity to $187.3 \pm 41.6 \%$ and $155.5 \pm 9.7 \%$ respectively, of AA control capacity. Stimulation of S3 dorsal root or S1-S3 ventral roots was not effective. Repeated stimulation of S1-S3 dorsal root did not induced a post-stimulation inhibition.

CONCLUSIONS-This study established a cat model of sacral neuromodualation of nociceptive bladder overactivity. The results revealed that the mechanisms underlying sacral neuromodulation are different for nociceptive and non-nociceptive bladder activity.

\section{Keywords}

bladder; cat; neuromodulation; overactive

\footnotetext{
*Correspondence Changfeng Tai, PhD, Department of Urology, University of Pittsburgh, 700 Kaufmann Building, Pittsburgh 15213, PA.m, cftai@pitt.edu.

Dr. Lori Birder led the peer-review process as the Associate Editor responsible for the paper. 


\section{1 | INTRODUCTION}

In the 1990s sacral neuromodulation was approved by the FDA for the treatment of refractory overactive bladder $(\mathrm{OAB}) .{ }^{1}$ It is now considered an effective treatment option for patients with severe refractory $\mathrm{OAB}$ symptoms after first line pharmacotherapy fails. ${ }^{2}$ However, the mechanisms of action underlying sacral neuromodulation therapy are still not fully understood. Development of an animal model of sacral neuromodulation is important for investigating the mechanisms underlying sacral neuromodulation of bladder activity.

Our previous study in anesthetized cats showed that electrical stimulation of the S1 or S2 sacral dorsal root inhibited non-nociceptive reflex bladder activity induced by saline distention of the bladder. ${ }^{3}$ However, our recent studies in cats indicate that pudendal/tibial neuromodulation of non-nociceptive or nociceptive reflex bladder activity involves different neurotransmitter mechanisms. ${ }^{4,5}$ Different mechanisms reflect the fact that different bladder afferent nerve fibers and neural pathways are activated during non-nociceptive or nociceptive reflex bladder activity. Saline distention of the bladder activates the nonnociceptive $\mathrm{A} \delta$ afferent fibers mediating a spinobulbospinal reflex pathway, ${ }^{6}$ while the nociceptive $\mathrm{C}$-fiber afferents are silent during saline distention. ${ }^{7}$ However, chemical irritation of the bladder can activate the nociceptive $\mathrm{C}$-fiber afferents mediating a spinal reflex pathway. ${ }^{8}$ Therefore, it is necessary to expand the study of sacral neuromodulation in cats to investigate reflex bladder overactivity induced by C-fiber afferents to examine the possibility that different mechanisms of sacral neuromodulation can exist under nociceptive bladder conditions.

In this study, dilute (0.5\%) AA was used to irritate the bladder, activate the nociceptive Cfiber afferents, and induced bladder overactivity in anesthetized cats. S1-S3 dorsal or ventral roots were electrically stimulated at different frequencies $(5-30 \mathrm{~Hz})$ to inhibit the bladder overactivity. The experimental model will be very useful for investigating neurotransmitter mechanisms involved in sacral neuromodulation of nociceptive bladder overactivity.

\section{2 | MATERIALS AND METHODS}

The experimental protocol involved in this study was approved by the Animal Care and Use Committee at the University of Pittsburgh.

\section{1 | Experimental setup}

A total of six cats (three female, three male, $3.1-4.1 \mathrm{~kg}$, Liberty Research Inc., Waverly, $\mathrm{NY}$ ) were used in this study. The animals were anesthetized by isoflurane (2-5\% in oxygen) during surgery and then maintained by $a$-chloralose anesthesia (initial $65 \mathrm{mg} / \mathrm{kg}$ intravenously with supplementation as needed) during data collection. The sensor of a pulse oximeter (9847 V, NONIN Medical, Inc., Plymouth, MN) was fixed on the tongue to monitor the heart rate and blood oxygen level. A tracheotomy was performed and a tube was inserted to maintain the airway open. A catheter was inserted into right carotid artery to monitor systemic blood pressure. Another catheter for intravenous infusion was placed into the right cephalic vein. Through an abdominal incision, the ureters were isolated, cut and drained externally with the distal ends of the cut ureters ligated to prevent leakage from the 
bladder. A double-lumen catheter was inserted into the bladder via a small cut in the proximal urethra and fixed in placed by a suture around the urethra. One lumen of the catheter was connected to a pump to slowly $(1-2 \mathrm{~mL} / \mathrm{min})$ infuse saline or $0.5 \%$ AA into the bladder and the other lumen was connected to a pressure transducer to record intravesical pressure.

The spinal cord and cauda equina were exposed between the L4 and S3 vertebrae via a dorsal laminectomy. The spinal dura was cut and the S1-S3 dorsal and ventral roots on the right side were separated for electrical stimulation. A bipolar stainless steel hook electrode was used during the experiment to stimulate individual dorsal/ventral roots by delivering monophasic electrical pulses ( $0.2 \mathrm{~ms}$ pulse width) that were generated by an electrical stimulator (S88, Grass Medical Instruments, Quincy, MA). The animal was mounted in a modified Narishige "Eccles" spinal cord frame in which the hip was supported by metal pins, and the spinous process at the rostral end of the laminectomy was secured with a clamp. The skin, cut mid-sagittally from L4 to S3, was tied along each margin to form a pool that was filled with warmed $\left(35-37^{\circ} \mathrm{C}\right)$ mineral oil. The temperature of the animal was maintained at $36-38^{\circ} \mathrm{C}$ using a heating pad during the experiments.

\section{2 | Stimulation protocol}

At the beginning of each experiment, multiple cystometrograms (CMGs) were performed with saline infusion to determine the bladder capacity that was defined as the bladder volume threshold to induce a bladder contraction of large amplitude $\left(>30 \mathrm{cmH}_{2} \mathrm{O}\right)$ and long duration (>20 s). Then, $0.5 \%$ AA was infused into the bladder to irritate the bladder, activate the nociceptive $\mathrm{C}$-fiber afferents, and induce bladder overactivity. Once the control bladder capacity stabilized during repeated AA CMGs, electrical stimulation was applied to the S1 dorsal root at the threshold $(T)$ intensity for inducing anal sphincter or toe twitching during the next three CMGs with stimulation frequency of 5, 15, or $30 \mathrm{~Hz}$ for each CMG. After the three stimulation CMGs, multiple (2-3) AA control CMGs were performed without stimulation to determine any post-stimulation effect. After testing the $\mathrm{S} 1$ dorsal root, the same CMG protocol was also used for S2 and then S3 dorsal root stimulation. The bladder was emptied after each CMG and a 5 min rest period was inserted between CMGs to allow the bladder reflex to recover.

Since $\mathrm{S} 1$ or $\mathrm{S} 2$ dorsal root stimulation at $5 \mathrm{~Hz}$ frequency was effective in inhibiting bladder overactivity and increasing bladder capacity, the $5 \mathrm{~Hz}$ stimulation at different intensities $(1 / 4,1 / 2$, or $1 T)$ was further tested during repeated AA CMGs to determine the minimal effective stimulation intensity. At the end of the experiment in two cats, the effect of sacral ventral root stimulation ( $\mathrm{S} 1, \mathrm{~S} 2$, or $\mathrm{S} 3$ ) was also examined during repeated AA CMGs with different stimulation frequencies $(5,15$, or $30 \mathrm{~Hz})$.

\section{3 | Data analysis}

The bladder capacity was measured from each CMG and normalized to the capacity measured during the first control CMG in each test group (i.e., different dorsal/ventral root stimulation). Repeated measurements in the same animal under the same conditions were averaged. The normalized data from different animals are presented as mean \pm standard 
error. Paired $t$-test was used to detect the significant reduction of bladder capacity induced by AA irritation. One-way ANOVA with Bonferroni correction followed by multiple comparisons of all pairs was used to determine the effects of stimulation frequency (Fig. 1) or intensity (Fig. 2) for an individual dorsal root (S1, S2, or S3) and to determine the effect of repeated dorsal root stimulation on control capacity (Fig. 3). Statistical significance is defined as $P<0.05$.

\section{3 | RESULT}

Intravesical infusion of $0.5 \%$ AA irritated the bladder, induced bladder overactivity, and significantly $(P<0.01)$ reduced bladder capacity to $62.6 \pm 11.7 \%$ of saline control capacity $(12.6 \pm 1.3 \mathrm{~mL})$. At threshold intensity for inducing anal sphincter or toe twitching, electrical stimulation of S1 or S2 dorsal roots at $5 \mathrm{~Hz}$ frequency inhibited bladder overactivity (Fig. 1A) and significantly $(P<0.05)$ increased bladder capacity to 187.3 $\pm 41.6 \%$ and $155.5 \pm 9.7 \%$, respectively, of AA control capacity ( $N=6$ cats, Fig. $1 \mathrm{~B}$ and C). Other stimulation frequencies ( 15 or $30 \mathrm{~Hz}$ ) did not significantly increase bladder capacity (Fig. 1B and C). S3 dorsal root stimulation had no effect on AA-induced bladder overactivity ( $N=6$ cats, Fig. 1D). Repeated stimulation of S1-S3 dorsal roots (three times for each root at 5,15 , and $30 \mathrm{~Hz}$ ) did not produce a post-stimulation inhibition, that is, the bladder capacity returned to the AA control capacity after the stimulation ( $N=6$ cats, Fig. $3)$.

In addition to the frequency, stimulation intensity was also critical for S1 or S2 dorsal root stimulation to be effective in inhibiting AA-induced bladder overactivity. At intensities below the motor threshold $(1 / 2$ or $1 / 4 T)$, the stimulation did not inhibit bladder overactivity and failed to significantly increase the bladder capacity ( $N=6$ cats, Fig. 2$)$.

In contrast to sacral dorsal root stimulation, preliminary tests in two cats (one male and one female) showed that electrical stimulation of S1, S2, or S3 ventral roots at motor threshold intensity and different frequencies $(5,15$, or $30 \mathrm{~Hz})$ had little effect on AA-induced bladder overactivity (Fig. 4).

\section{4 | DISCUSSION}

This study in anesthetized cats showed that $\mathrm{S} 1$ or $\mathrm{S} 2$ dorsal root stimulation was effective in inhibiting bladder overactivity induced by $0.5 \%$ AA irritation of the bladder (Fig. 1A-C), while electrical stimulation of S3 dorsal root (Fig. 1D) was not effective. The inhibition occurred at a stimulation frequency of $5 \mathrm{~Hz}$ but not $15 \mathrm{or} 30 \mathrm{~Hz}$ (Fig. 1A-C) and required a minimal stimulation intensity at the threshold for evoking reflex twitching of the anal sphincter or toe (Fig. 2). Repeated dorsal root stimulation did not induce a post-stimulation inhibitory effect (Fig. 3). These results established a cat model that will be useful for examining the mechanisms underlying sacral neuromodulation of irritation-induced bladder overactivity.

Previous studies in cats ${ }^{3}$ have shown that S1/S2 dorsal root stimulation is effective in inhibiting non-nociceptive bladder activity induced by saline distention. The current study further shows that the minimal stimulation intensities required to induce the inhibition under 
both non-nociceptive and nociceptive conditions are similar, indicating that a similar group of afferent nerve fibers in the S1/S2 dorsal roots is involved under both conditions. However, repeated stimulation of the same afferent nerves in the dorsal roots produced significant post-stimulation inhibition of non-nociceptive bladder activity ${ }^{3}$ but no post-stimulation inhibition of nociceptive bladder overactivity (Fig. 3), indicating that nociceptive afferent input from the bladder can either block the post-stimulation inhibition or activate a reflex mechanism that is resistant to post-stimulation inhibition. Similar post-stimulation inhibition was also observed for tibial neuromodulation in cats. ${ }^{9}$ The clinical implications of these results could be that those patients responding to tibial neuromodulation might have OAB caused by the overactivity in the supraspinal micturition pathway because clinical tibial neuromodulation utilizes a post-stimulation effect. Meanwhile, those patients not responding to tibial neuromodulation might be better treated by pudendal or sacral neuromodulation that stimulates the nerve continuously and does not depend on a post-stimulation effect. It is worth noting that in acute animal studies the post-stimulation inhibition which lasted more than $2 \mathrm{~h}^{9}$ may not be comparable to the clinical post-stimulation inhibition produced by chronic tibial neuromodulation that lasts for several weeks. Chronic animal studies are needed in order to determine the full duration of post-stimulation inhibition observed in recent animal studies. 3,9

The different results of post-stimulation inhibition also indicate that the mechanisms underlying sacral neuromodulation of non-nociceptive and nociceptive bladder activity could be very different. This idea is also supported by our previous studies in cats showing that opioid receptors are involved in tibial neuromodulation only under a nociceptive condition but not under a non-nociceptive condition ${ }^{4}$ and the involvement of spinal $\mathrm{GABA}_{\mathrm{A}}$ receptors in pudendal neuromodulation is also observed only under a nociceptive bladder condition. ${ }^{5}$ It is worth noting that a previous study in cats ${ }^{10}$ showed that picrotoxin $\left(\mathrm{GABA}_{\mathrm{A}}\right.$ receptor antagonist) at a very high dose ( $1.5 \mathrm{mg} / \mathrm{kg}$ i.v.) removed pudendal inhibition of isovolumetric bladder contractions induced by saline distention. However, due to the convulsant effect of picrotoxin at this high dose that can significantly increase reflex excitability and reduce bladder capacity, it is very difficult to conclude that the effects of picrotoxin are selective and that $\mathrm{GABA}_{\mathrm{A}}$ receptors are involved in pudendal inhibition of non-nociceptive bladder activity.

Sacral neuromodulation could activate afferent fibers from both tibial and pudendal nerves that travel in the sacral dorsal roots. Our previous study in cats has shown that poststimulation inhibition can be induced by tibial nerve stimulation only during saline infusion (non-nociceptive condition) but not during AA irritation (nociceptive condition), ${ }^{9}$ while pudendal nerve stimulation cannot induce a post-stimulation effect under either bladder condition. ${ }^{11}$ These comparative results indicate that the afferent nerve fibers from the tibial nerve that travel in sacral dorsal roots ${ }^{12}$ might be responsible for the post-stimulation inhibition elicited by sacral dorsal root stimulation under non-nociceptive bladder condition. ${ }^{3}$ However, pudendal afferent nerves also travel in sacral dorsal roots. ${ }^{13}$ Our previous studies in cats show that pudendal nerve stimulation produces bladder inhibition at low frequency $(5 \mathrm{~Hz})$ but not at high frequency $(15-30 \mathrm{~Hz}),{ }^{14}$ while tibial nerve stimulation is effective at both low and high frequencies $(5-30 \mathrm{~Hz}) .{ }^{15}$ The frequency similarity between pudendal and sacral dorsal root stimulation (see Fig. 1) suggests that pudendal afferent 
nerves in sacral dorsal roots may also be activated. Additional investigation is warranted to determine the different contributions of pudendal and tibial afferent nerves to the inhibition of bladder activity induced by sacral dorsal root stimulation.

Significant species differences exist in sacral neuromodulation. ${ }^{3,16-25}$ In humans electrical stimulation is effective when applied unilaterally to S3 spinal root at an intensity slightly below the motor threshold. ${ }^{16}$ However, in rats bilateral stimulation of lumbar/sacral spinal roots is needed to inhibit bladder activity. ${ }^{17-21}$ Bilateral stimulation of S1 spinal root in rats inhibits non-nociceptive bladder activity at an intensity greater than 12 times motor threshold, ${ }^{18}$ while bilateral stimulation of L6 spinal root requires an intensity greater than two times motor threshold. ${ }^{19}$ Stimulation at the motor threshold intensity in rats can only produce inhibition under non-nociceptive conditions when both L6 and S1 spinal roots are stimulated bilaterally, ${ }^{17}$ but under nociceptive conditions bilateral stimulation of either the L6 or S1 spinal roots can elicit inhibition. ${ }^{24,25}$ On the other hand, in humans and larger animals including cats, ${ }^{3,22}$ pigs, ${ }^{23}$ and sheep ${ }^{24}$ unilateral stimulation of a single sacral spinal root can inhibit bladder activity; and in cats this occurs at the motor threshold for the S1 or S2 dorsal roots under a non-nociceptive ${ }^{3}$ as well as nociceptive conditions (the current study). In sheep unilateral stimulation of S3 spinal root was only investigated under a nonnociceptive condition, ${ }^{24}$ and in pigs unilateral stimulation of S3 spinal root was only studied under nociceptive conditions. ${ }^{23}$ Because stimulation parameters and the location of stimulation as well as experimental conditions have been subjected to a detailed analysis in the cat, the cat model is probably most suited for future comparative studies of the mechanisms underlying the effects of sacral neuromodulation on non-nociceptive and nociceptive bladder activity.

Our previous studies in cats ${ }^{3}$ have shown that stimulation of sacral ventral roots has no effect on bladder activity under non-nociceptive conditions. Our preliminary tests in two cats show a similar result under nociceptive conditions (Fig. 4). These results in cats agree with previous clinical studies showing that the therapeutic effects of sacral neuromodulation are due to stimulation of afferent nerve fibers instead of efferent nerve fibers in the spinal roots. ${ }^{25}$ Our previous study in cats ${ }^{5}$ also showed that the sympathetic inhibitory pathway to the bladder passing through the hypogastric nerves did not play a role in sacral neuromodulation of non-nociceptive bladder activity. However, our recent study in cats ${ }^{26}$ has shown that pudendal neuromodulation can activate the sympathetic (hypogastric) efferent output to inhibit the bladder activity via a $\beta$-adrenergic mechanism only under a nociceptive bladder condition but not under a non-nociceptive bladder condition. Since sacral dorsal root stimulation probably activates the pudendal afferent fibers, additional studies are suggested to further determine the role of hypogastric nerves in sacral neuromodulation of nociceptive bladder overactivity.

Clinical application of sacral neuromodulation often uses stimulation frequencies of 10 or 15 Hz. ${ }^{19}$ However, our studies in cats (Fig. 1$)^{3}$ show that low frequency $(5 \mathrm{~Hz})$ sacral neuromodulation is better than high frequency $(15 \mathrm{~Hz})$ for inhibiting bladder activity. Previous studies in cats ${ }^{22}$ also show that $7-10 \mathrm{~Hz}$ is the optimal frequency for sacral neuromodulation. A recent study in humans ${ }^{16}$ further indicates that sacral neuromodulation at $5.2 \mathrm{~Hz}$ can produce the same efficacy as higher frequencies $(10,21$, or $40 \mathrm{~Hz})$. Therefore, 
both animal and human studies suggest that a lower frequency $(5 \mathrm{~Hz})$ should be used in clinical application because it can significantly reduce the consumption of electricity and save the battery life of the implanted stimulator.

With the minimal number of cats per gender $(N=3)$, this study did not detect any statistically significant difference between male and female animals. However, as a group ( $N$ $=6$ cats for both genders) statistically significant effects of sacral neuromodulation were detected (Figs. 1 and 2), indicating that sex difference was not a major factor in this study. Certainly, using the sacral neuromodulation model established in this study and a larger number of cats per gender it might be possible to detect a sex difference if it exists. More studies aimed at sex difference in sacral neuromodulation are warranted.

The frequency effects were determined by stimulating different dorsal roots in a sequence (S1, S2, and then S3). This experimental order may cause a weaker effect for the tests performed at a later time. However, this seems unlikely to be a factor because: (1). The control CMGs did not change with time (Fig. 3); (2) Data in Figure 2 were obtained after repeated stimulation of S1, S2, and S3 dorsal roots, but the same extent of inhibition was still obtained by S1 or S2 dorsal root stimulation at 1T intensity (compare data in Figs. 1 and 2).

In summary, this study in cats established a large animal model of sacral neuromodualation of nociceptive bladder overactivity. It will be very useful for future comparative studies to understand the different mechanisms underlying sacral neuromodulation of non-nociceptive and nociceptive bladder activity. Understanding the mechanisms of sacral neuromodulation could further improve this FDA-approved therapy or discover new therapies for OAB.

\section{Acknowledgments}

This study is supported by the National Institutes of Diabetes and Digestive and Kidney Diseases under grants DK-094905, DK-102427, and DK-091253.

Funding Information

This work was supported by National Institutes of Diabetes and Digestive and Kidney Diseases DK-094905 and DK-102427, and DK-091253.

\section{References}

1. Schmidt RA, Jonas U, Oleson KA, et al. Sacral nerve stimulation for treatment of refractory urinary urge incontinence. Sacral Nerve Stimulation Study Group. J Urol. 1999; 162:352-357. [PubMed: 10411037]

2. Gormley EA, Lightner DJ, Faraday M, Vasavada SP. Diagnosis and treatment of overactive bladder (non-neurogenic) in adults: AUA/ SUFU guideline amendment. J Urol. 2015; 193:1572-1580. [PubMed: 25623739]

3. Zhang F, Zhao S, Shen B, et al. Neural pathways involved in sacral neuromodulation of reflex bladder activity in cats. Am J Physiol Renal Physiol. 2013; 304:F710-F717. [PubMed: 23283999]

4. Tai C, Larson JA, Ogagan PD, et al. Differential role of opioid receptors in tibial nerve inhibition of nociceptive and nonnociceptive bladder reflexes in cats. Am J Physiol Renal Physiol. 2012; 302:F1090-F1097. [PubMed: 22237803] 


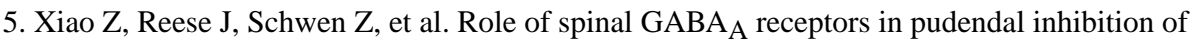
nociceptive and nonnociceptive bladder reflexes in cats. Am J Physiol Renal Physiol. 2014; 306:F781-F789. [PubMed: 24523385]

6. Fowler CJ, Griffiths D, de Groat WC. The neural control of micturition. Nat Rev Neurosci. 2008; 9:453-466. [PubMed: 18490916]

7. Habler HJ, Jänig W, Koltzenburg M. Activation of unmyelinated afferent fibres by mechanical stimuli and inflammation of the urinary bladder in the cat. J Physiol. 1990; 425:545-562. [PubMed: 2213588]

8. Xiao Z, Rogers MJ, Shen B, et al. Somatic modulation of spinal reflex bladder activity mediated by nociceptive bladder afferent nerve fibers in cats. Am J Physiol Renal Physiol. 2014; 307:F673F679. [PubMed: 25056352]

9. Ferroni MC, Slater RC, Shen B, et al. Role of the brain stem in tibial inhibition of the micturition reflex in cats. Am J Physiol Renal Physiol. 2015; 309:F242-FF50. [PubMed: 26017973]

10. McGee MJ, Danziger ZC, Bamford JA, Grill WM. A spinal GABAergic mechanism is necessary for bladder inhibition by pudendal afferent stimulation. Am J Physiol Renal Physiol. 2014; 307:F921-F930. [PubMed: 25143456]

11. Matsuta Y, Schwen Z, Mally AD, et al. Effect of methysergide on pudendal inhibition of micturition reflex in cats. Exp Neurol. 2013; 247:250-258. [PubMed: 23688680]

12. Vanderhorst VG, Holstege G. Organization of lumbosacral motoneuronal cell groups innervating hindlimb, pelvic floor, and axial muscles in the cat. J Comp Neurol. 1997; 382:46-76. [PubMed: 9136811]

13. Thor KB, Morgan C, Nadelhaft I, Houston M, De Groat WC. Organization of afferent and efferent pathways in the pudendal nerve of the female cat. J Comp Neurol. 1989; 288:263-279. [PubMed: 2477417]

14. Tai C, Chen M, Shen B, et al. Plasticity of urinary bladder reflexes evoked by stimulation of pudendal afferent nerves after chronic spinal cord injury in cats. Exp Neurol. 2011; 228:109-117. [PubMed: 21192927]

15. Tai C, Chen M, Shen B, Wang J, Roppolo JR, de Groat WC. Irritation induced bladder overactivity is suppressed by tibial nerve stimulation in cats. J Urol. 2011; 186:326-330. [PubMed: 21600604]

16. Marcelissen TA, Leong RK, Nieman FH, de Bie RA, van Kerrebroeck PE, de Wachter SG. The effect of pulse rate changes on the clinical outcome of sacral neuromodulation. J Urol. 2011; 185:1781-1785. [PubMed: 21420118]

17. Dieter AA, Degoski DJ, Dolber PC, Fraser MO. The effects of bilateral bipolar sacral neurostimulation on urinary bladder activity during filling before and after irritation in a rat model. Neurourol Urodyn. 2015; 34:387-391. [PubMed: 24802624]

18. Riazimand SH, Mense S. A rat model for studying effects of sacral neuromodulation on the contractile activity of a chronically inflamed bladder. BJU Int. 2004; 94:158-163. [PubMed: 15217453]

19. Su X, Nickles A, Nelson DE. Neuromodulation in a rat model of the bladder micturition reflex. Am J Physiol Renal Physiol. 2012; 302:F477-F486. [PubMed: 22049401]

20. Su X, Nickles A, Nelson DE. Optimization of neuromodulation for bladder control in a rat cystitis model. Neuromodulation. 2016; 19:101-107. [PubMed: 26517575]

21. Wang Y, Zhou Y, Mourad MS, Hassouna MM. Neuromodulation reduces urinary frequency in rats with hydrochloric acid-induced cystitis. BJU Int. 2000; 86:726-730. [PubMed: 11069385]

22. Schultz-Lampel D, Jiang C, Lindstrom S, Thuroff JW. Experimental results on mechanisms of action of electrical neuromodulation in chronic urinary retention. World J Urol. 1998; 16:301-304. [PubMed: 9833307]

23. Kaufmann S, Naumann CM, Hamann MF, et al. Unilateral vs bilateral sacral neuromodulation in pigs with formalin-induced detrusor hyperactivity. BJU Int. 2009; 103:260-263. [PubMed: 18710441]

24. Brink TS, Zimmerman PL, Mattson MA, Su X, Nelson DE. A chronic, conscious large animal platform to quantify therapeutic effects of sacral neuromodulation on bladder function. J Urol. 2015; 194:252-258. [PubMed: 25661299] 
25. Kessler TM, La Framboise D, Trelle S, et al. Sacral neuromodulation for neurogenic lower urinary tract dysfunction: Systematic review and meta-analysis. Eur Urol. 2010; 58:865-874. [PubMed: 20934242]

26. Brian TK, Timothy DL, Zhaocun Z, et al. Sympathetic $\beta$-adrenergic mechanism in pudendal inhibition of nociceptive and non-nociceptive reflex bladder activity. Am J Physiol Renal Physiol. 2016; 311:F78-F84. [PubMed: 27170683] 

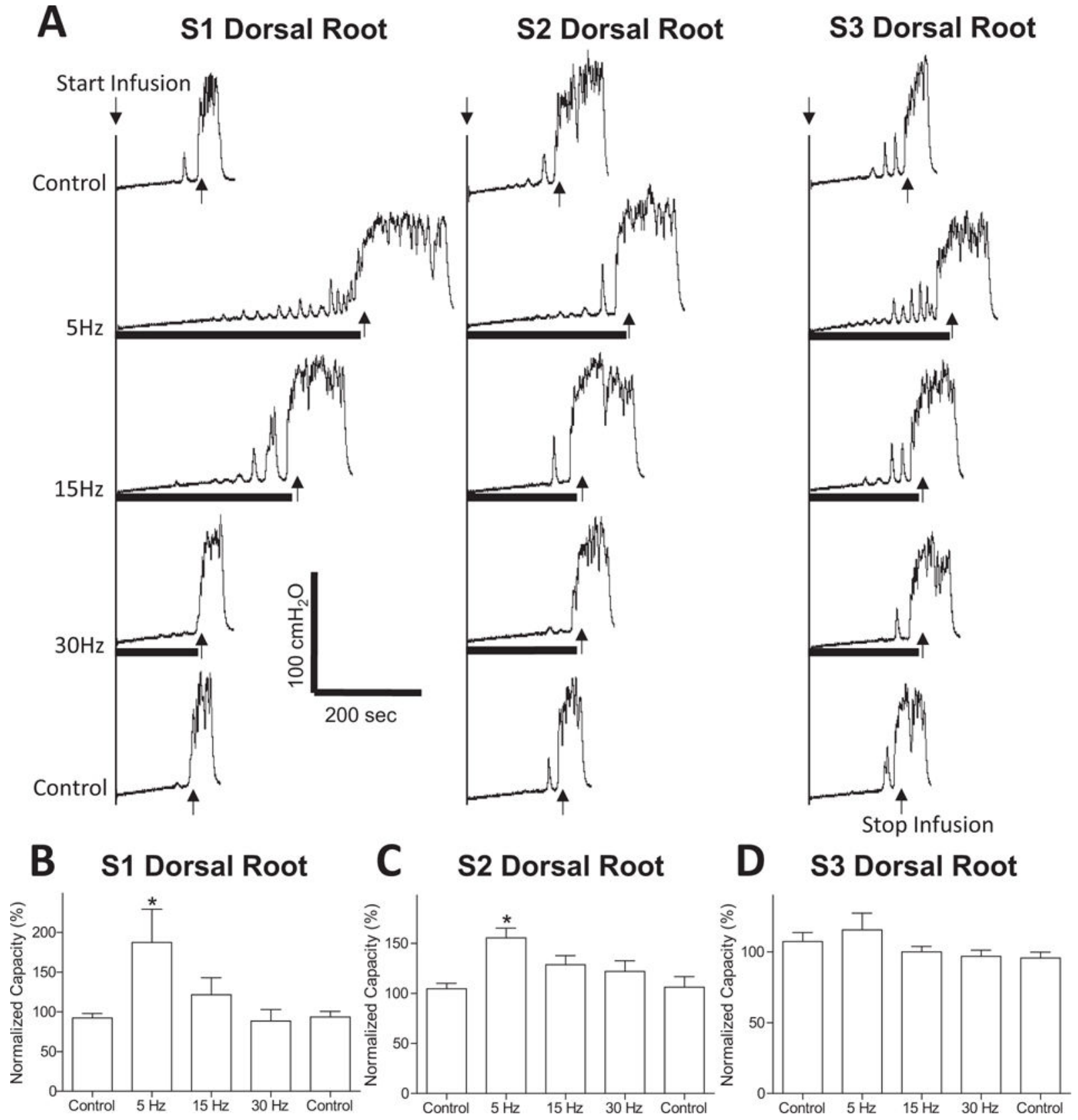

FIGURE 1.

Inhibition of AA irritation-induced bladder overactivity by stimulation of sacral dorsal roots S1-S3. (A) Stimulation at threshold ( $T$ ) intensity and different frequencies $(5,15,30 \mathrm{~Hz})$ was applied during repeated CMGs. The black bar under bladder pressure trace indicates the stimulation duration. $T=0.2 \mathrm{~V}$ for S1 and $\mathrm{S} 2, T=6 \mathrm{~V}$ for S3. (B-D) Normalized bladder capacity measured during repeated CMGs with S1 (B), S2 (C), or S3 (D) dorsal root stimulation at different frequencies. *Indicates significantly different from the first control $(P<0.05, N=6$, one-way ANOVA). 
A

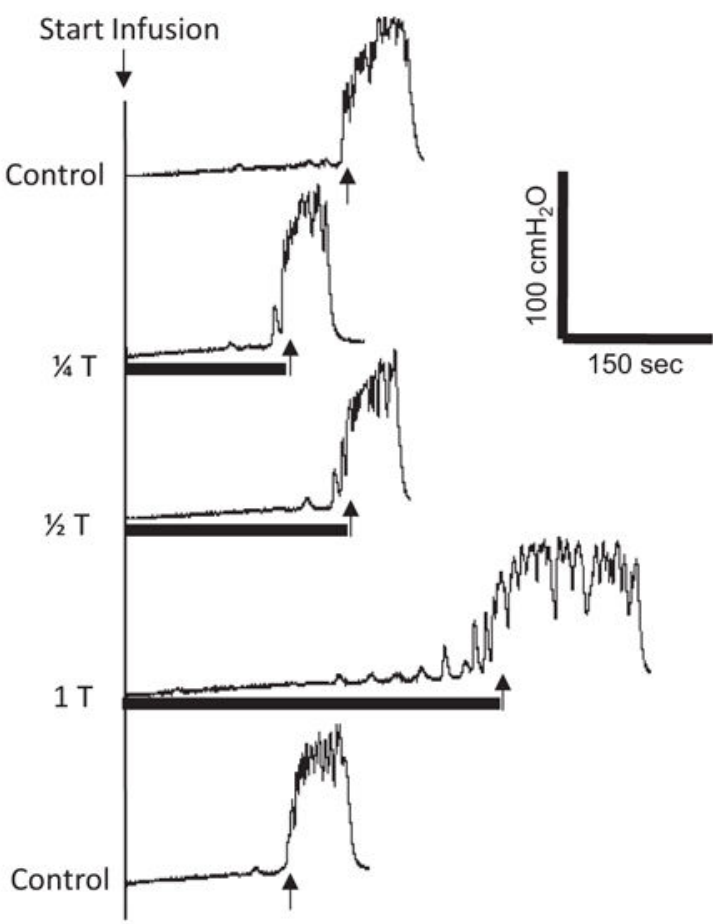

S2 Dorsal Root

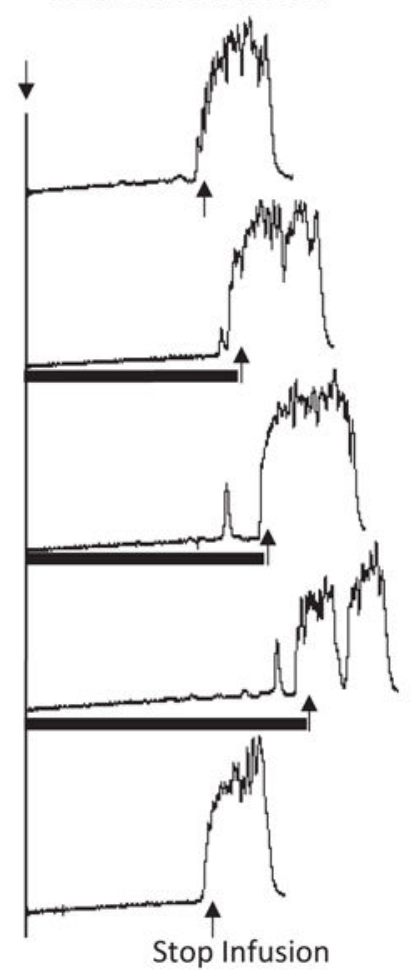

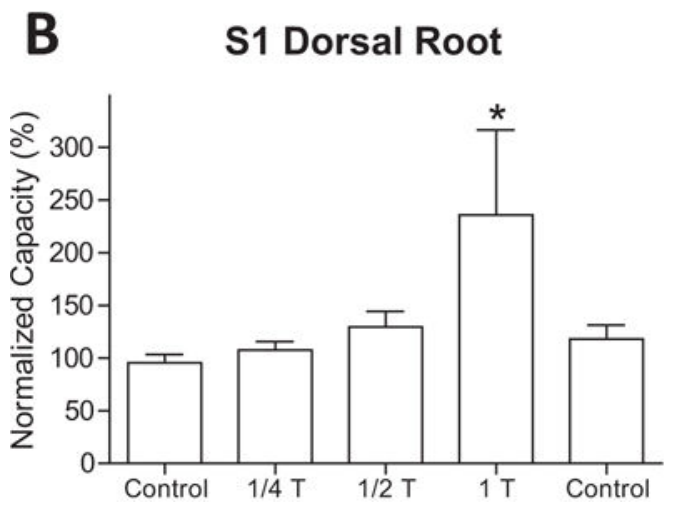

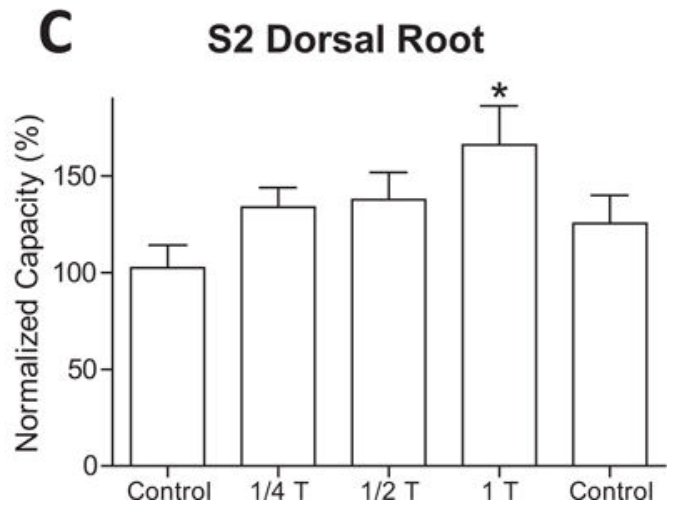

FIGURE 2.

Effect of different stimulation intensities on S1/S2 dorsal root inhibition of bladder overactivity induced by AA irritation. (A) Stimulation $(5 \mathrm{~Hz})$ at different intensities relative to the threshold $(T)$ intensity for evoking reflex twitching of anal sphincter or toe was applied during repeated CMGs. The black bar under bladder pressure trace indicates the stimulation duration. $T=0.2 \mathrm{~V}$ for $\mathrm{S} 1$ and $\mathrm{S} 2$. (B-C) Normalized bladder capacity measured during repeated CMGs with S1 (B) or S2 (C) dorsal root stimulation at different intensities. *Indicates significantly different from the first control ( $P<0.05, N=6$, one-way ANOVA). 


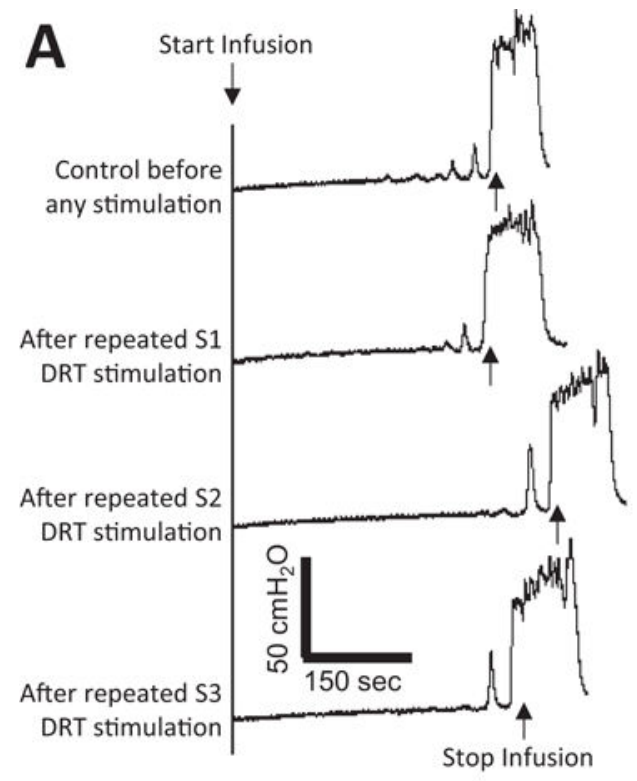

B

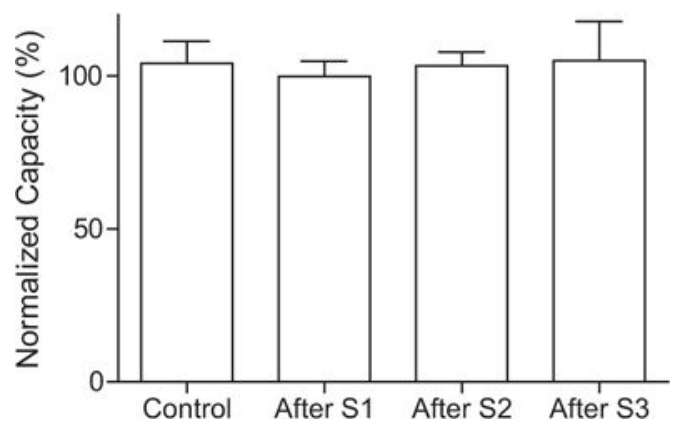

FIGURE 3.

Repeated stimulation of sacral dorsal root (DRT) S1-S3 did not produce post-stimulation inhibition of bladder overactivity induced by AA irritation. (A) Control CMGs before and after repeated (three times at 5, 15, and $30 \mathrm{~Hz}$ ) S1, S2, or S3 DRT stimulation (2-10 min each time). (B) Bladder capacity was not changed after repeated S1, S2, and S3 DRT stimulation. 
A S1 Ventral Root

S2 Ventral Root

S3 Ventral Root
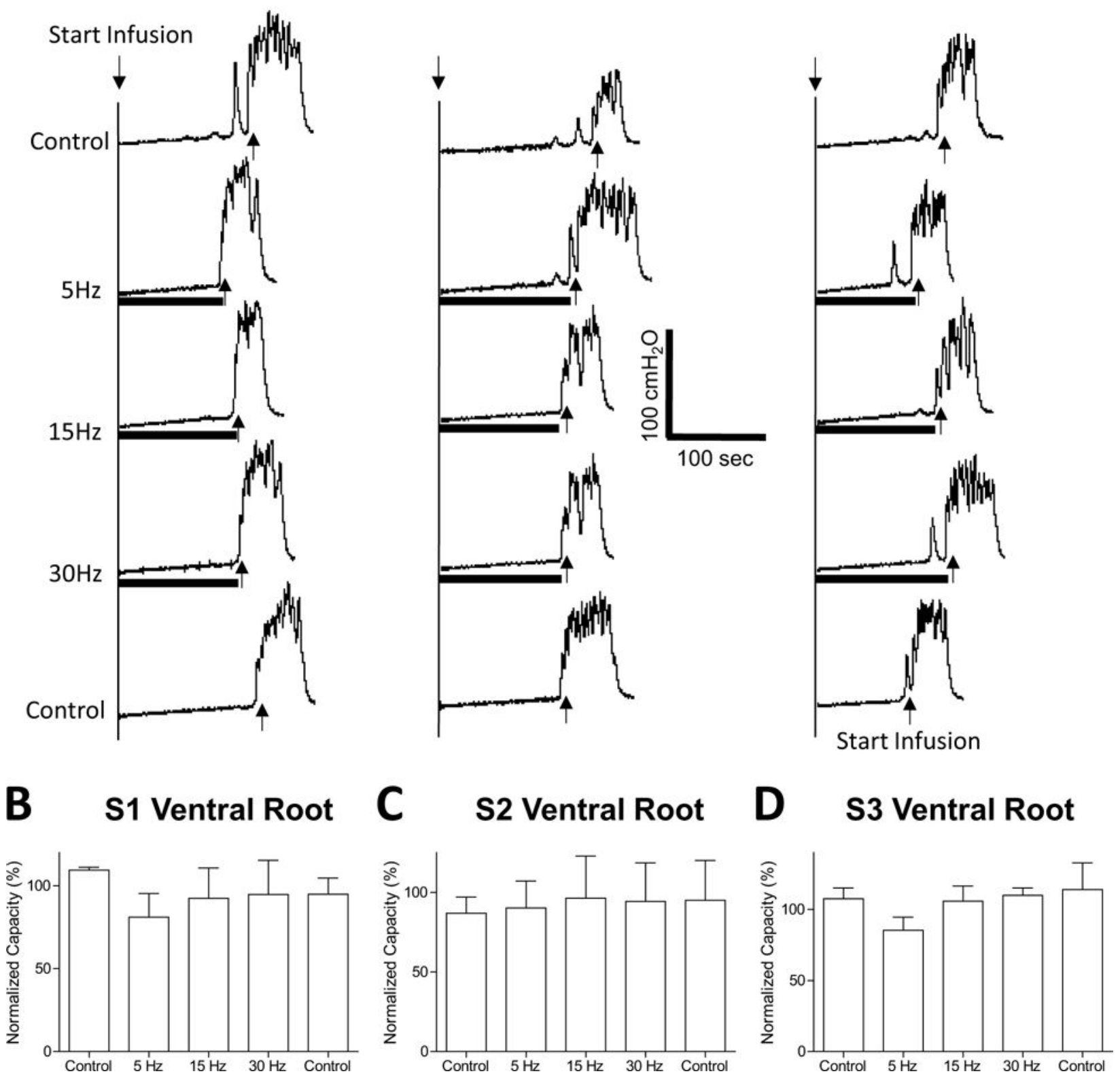

FIGURE 4.

Stimulation of S1-S3 ventral roots did not inhibit reflex bladder activity or increase bladder capacity during AA irritation. (A) Stimulation at threshold ( $T$ ) intensity and different frequencies $(5,15,30 \mathrm{~Hz})$ was applied during repeated CMGs. The black bar under bladder pressure trace indicates the stimulation duration. $T=0.15 \mathrm{~V}$ for $\mathrm{S} 1, T=0.07 \mathrm{~V}$ for $\mathrm{S} 2$, and $T$ $=0.2 \mathrm{~V}$ for S3. (B-D) Normalized bladder capacity measured during repeated CMGs with S1 (B), S2 (C), or S3 (D) ventral root stimulation at different frequencies. 